In conclusion, we accept the writers' assertion that CAV is not a direct measure of spinal sudomotor velocity. We do consider, however, that it is a reflection of that velocity and has the advantage over other methods that it should be obtainable in all normal subjects. Pathological disturbances at spinal level may prove to be reflected in CAV values either bilaterally or unilaterally in patient populations".

Department of Neurology and

Serge Bajada

Clinical Neurophysiology,

Fremantle Hospital,

and

Fremantle, WA 6160 (Australia)

and

Department of Neurology,

Mayo Clinic,

Rochester, MN 55905 (U.S.A.)

Wally Knezevic

\title{
Answer to Letter of S. Bajada and W. Knezevic
}

Dear Editors,

Knezevic and Bajada (1985) reported measurement of the peripheral autonomic surface potential (PASP) and demonstrated its application in evaluating patients with suspected autonomic neuropathy. A similar procedure (Willems and Albers 1980) has been used in our laboratory in the routine evaluation of autonomic dysfunction. Our normal values are in agreement with those reported by Knezevic and Bajada with the exception of the PASP amplitudes. Following three conditioning stimuli, we average the subsequent three PASPs to obtain amplitude and latency measurements for upper and lower extremities. Because of variability in the initial negative deflection, we record both the baseline to the maximum positive peak and peak to peak amplitudes. Control amplitude values (baseline to positive peak) average $1.9 \mathrm{mV}$ (range $0.6-10.0 \mathrm{mV}$ ) for the hand and $1.3 \mathrm{mV}$ (range $0.3-6.2 \mathrm{mV}$ ) for the foot for subjects aged 28-77 years. Peak to peak amplitudes, while more variable, average $2.8 \mathrm{mV}$ for the hand and $2.4 \mathrm{mV}$ for the foot. These values are substantially greater than those reported by Knezevic and Bajada $(0.48 \mathrm{mV}$ and $0.1 \mathrm{mV}$ for the palmar and plantar responses, respectively). This difference is likely technical, related to the filter settings used. The characteristic biphasic PASP is approximately sinusoidal and has a full cycle duration of up to $3 \mathrm{~s}$, the majority of responses having a frequency of less than $0.5 \mathrm{~Hz}$. We use bandpass filter settings of 0.1 and $75 \mathrm{~Hz}$. Equivalent settings are available on most conventional electromyography equipment. Knezevic and Bajada, however, have reported bandpass filter settings of $2 \mathrm{~Hz}$ and $5 \mathrm{kHz}$. Although these are traditional settings for motor conduction studies, such settings substantially reduce the amplitude of any signal having a frequency lower than the $2 \mathrm{~Hz}$ cut-off frequency. Their reported technique therefore discards much of the evoked response, greatly reducing all amplitude measurements. Our experience indicates that response amplitude is of greater importance in defining abnormalities of autonomic function than the latency measurements. It is therefore important to use filter settings that do not inadvertently minimize the predominant low frequency component of this signal.

\section{REFERENCES}

Knezevic, W. and S. Bajada (1985) Peripheral autonomic surface potential - A quantitative technique for recording sympathetic conduction in man, J. Neurol. Sci., 67: 239-251.

Willems, W.J. and J.W. Albers (1980) Evoked autonomic responses in Shy-Drager syndrome (Abstr.), Muscle \& Nerve, 3: 185.

Department of Neurology,

James W. Albers

University of Michigan Medical Center,

1405 East Ann Street,

Ann Arbor, MI 48109 (U.S.A.) 